\title{
Long-term safety, efficacy, and patient acceptability of the tulobuterol patch
}

This article was published in the following Dove Press journal:

Research and Reports in Transdermal Drug Delivery

29 April 2013

Number of times this article has been viewed

\author{
Tomohiro Ichikawa' \\ Hisatoshi Sugiura ${ }^{2}$ \\ 'Third Department of Internal \\ Medicine, Wakayama Medical \\ University, School of Medicine, \\ Wakayama, ${ }^{2}$ Department of \\ Respiratory Medicine, Tohoku \\ University Graduate School \\ of Medicine, Sendai, Japan
}

\begin{abstract}
The tulobuterol patch contains a $\beta_{2}$-adrenergic agonist, is the first transdermal bronchodilator, and was developed in Japan. It relies on a matrix-type transdermal delivery system that contains both crystallized and molecular forms of tulobuterol. This delivery system enables the drug content to be released steadily over 24 hours. When the tulobuterol patch is applied to the skin at bedtime, the serum concentration of tulobuterol peaks in the early morning, suppressing the morning dip in pulmonary function. This pharmacological property also prevents steep increases in plasma drug levels, which reduces the risk of adverse events. Because the tulobuterol patch is easy to use and requires only once-daily application, treatment adherence in patients using the patch is superior to that of other therapies. The clinical efficacy of the patch in patients with asthma and chronic obstructive pulmonary disease at any age has been established in many clinical studies since launch in 1998, and the product has a good safety profile. Therefore, the tulobuterol patch is currently used for the long-term treatment of patients with asthma and chronic obstructive pulmonary disease. Here we review the characteristics of the tulobuterol patch and the results of clinical trials that have investigated the effects of the patch on pulmonary function, quality of life, and adherence. We also discuss the usefulness of the tulobuterol patch in clinical practice.
\end{abstract}

Keywords: tulobuterol patch, long-acting $\beta_{2}$-agonist, crystal reservoir system, chronotherapy, asthma, chronic obstructive pulmonary disease

\section{Introduction}

The tulobuterol patch was developed in Japan, and is the first bronchodilator available as an adhesive transdermal patch containing a $\beta_{2}$-adrenergic agonist. The patch was designed for use as chronotherapy for nocturnal asthma when applied at bedtime, ${ }^{1,2}$ and is currently used in the long-term management of asthma and chronic obstructive pulmonary disease (COPD) in Japan, Korea, and People's Republic of China. The patch is applied to the skin once daily, continuously releases its active ingredient, and maintains effective serum tulobuterol concentrations for 24 hours. Therefore, the tulobuterol patch provides long-acting $\beta$-agonist activity, despite tulobuterol being categorized pharmacokinetically as a short-acting $\beta_{2}$-agonist. Because the tulobuterol patch requires only once-daily application, treatment adherence is excellent. Further, its clinical efficacy and safety in the treatment of chronic airways disease have been established since its launch in 1998. In this review, we describe the characteristics of the tulobuterol patch and provide evidence of its long-term efficacy in asthma and COPD. We also discuss the safety and patient acceptability of the patch.
Correspondence: Hisatoshi Sugiura Department of Respiratory Medicine, Tohoku University Graduate School of Medicine, I-I Seiryo-machi, Aoba-ku, Sendai 980-8574, Japan $\mathrm{Tel}+8|227| 78539$ Fax +81227178549

Email sugiura@rm.med.tohoku.ac.jp submit your manuscript | www.dovepress.com

Dovepress

http://dx.doi.org// 0.2 | 47/RRTD.S3403 |
Research and Reports in Transdermal Drug Delivery 2013:2 9-18

(C) 2013 Ichikawa and Sugiura, publisher and licensee Dove Medical Press Ltd. This is an Open Access article which permits unrestricted noncommercial use, provided the original work is properly cited. 


\section{Tulobuterol patch formulation}

Nocturnal worsening of asthma, referred to more simply as "nocturnal asthma", is a common and important problem for asthmatic patients. A survey of 7729 patients with varying severity of asthma showed that $74 \%$ of them awoke at night at least once a week, and $64 \%$ awoke at least three times a week because of asthma symptoms. ${ }^{3}$ Nocturnal asthma is defined as variable exacerbation of the underlying asthma condition, which is associated with increased symptoms of airway hyper-responsiveness and/or worsening of lung function, and a need for medication. ${ }^{4}$ These changes are associated with circadian variations in lung function. Lung function measured by peak expiratory flow or forced expiratory volume in one second $\left(\mathrm{FEV}_{1}\right)$ in a healthy individual varies according to the circadian rhythm, with maximal lung function occurring at about $4 \mathrm{pm}$ and minimal lung function occurring near 4 am. .,6 $^{5}$ Compared with the normal range of variation $(5 \%-8 \%)$ of lung function in healthy subjects, nocturnal asthma exaggerates the changes in pulmonary function by more than $15 \%$. This decrease in lung function in the early morning as a result of nocturnal asthma is known as the "morning dip". Chronotherapy based on the circadian rhythm is needed in asthmatics to prevent this morning dip and to improve quality of life. The tulobuterol patch was initially developed to prevent the morning dip and to sustain drug efficacy over 24 hours (Figure 1). To accomplish this, a matrixtype transdermal delivery system was used for formulation of the tulobuterol patch. The branded patch (Hokunalin ${ }^{\circledR}$ Tape, Abbott Japan Co, Ltd, Tokyo, and Maruho Co, Ltd, Osaka, Japan) contains both crystallized and molecular forms of tulobuterol in an adhesive layer. It also includes a controlled drug release mechanism known as the crystal reservoir system (Figure 2). After the tulobuterol patch is applied to the skin, the molecular tulobuterol is gradually absorbed percutaneously. As the number of tulobuterol molecules in the patch decreases, additional molecules are supplied by dissociation of the tulobuterol crystals into a molecular and absorbable form. This mechanism enables timed release of tulobuterol, which means that the drug concentration can peak when the symptoms of asthma are at their worst. Because currently available oral $\beta_{2}$-agonists, such as tulobuterol, salbutamol, terbutaline, and bambuterol, reach peak levels in serum within 3 hours of administration, patients with asthma who take these medications before bedtime cannot achieve maximum bronchodilation when most needed during the morning dip. In addition, oral $\beta_{2}$-agonists often have clinically significant systemic adverse effects, such as tremor and palpitations, due to steep increases in plasma drug levels. ${ }^{7,8}$ The tulobuterol patch provides gradual and continuous drug release, so reduces the risk of systemic adverse effects caused by high plasma drug concentrations.

\section{Pharmacokinetics}

Uematsu et al studied the pharmacokinetics of tulobuterol given transdermally or by aerosol inhalation in healthy male volunteers. ${ }^{9}$ When the $2 \mathrm{mg}$ tulobuterol patch was administered to healthy adults, it was found to be well absorbed, with an absorption lag-time of about four hours. Serum tulobuterol concentrations peaked at 9-12 hours and

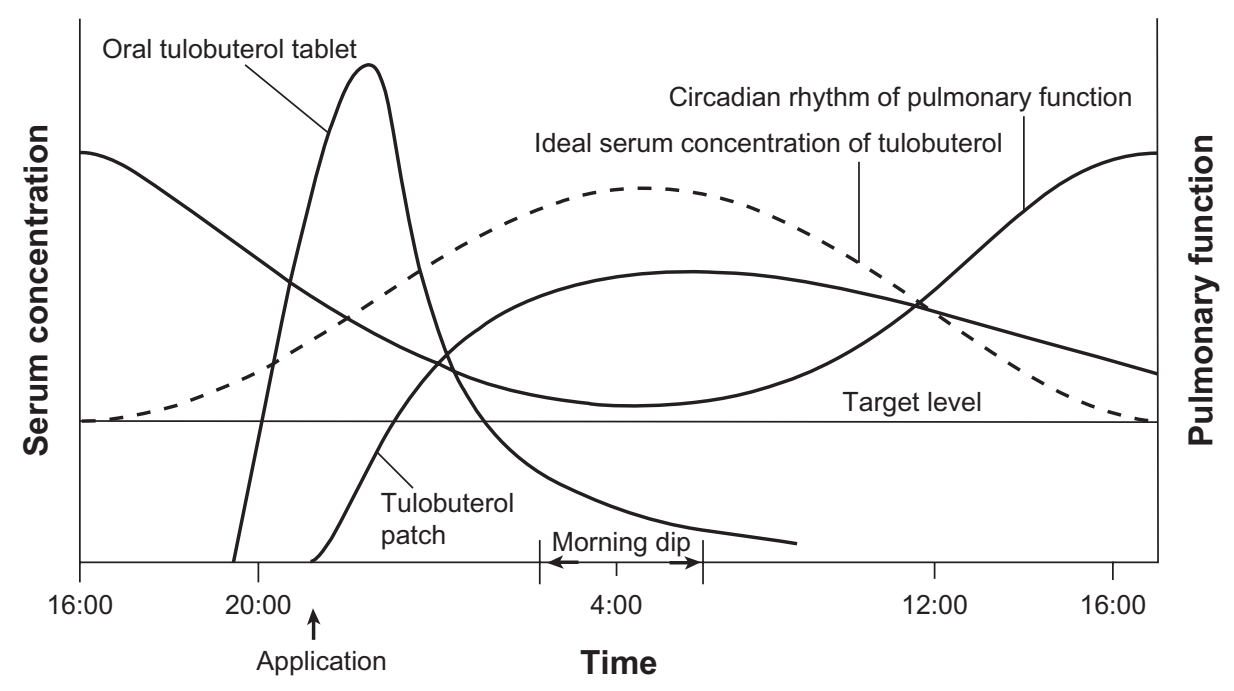

Figure I Time profiles for serum concentration of tulobuterol.

Notes: Dashed line indicates the ideal time profile of the serum concentration of tulobuterol on the basis of circadian pulmonary function (line). When the tulobuterol patch is applied before bedtime, the maximum concentration of tulobuterol is achieved during the morning dip (bold line). 


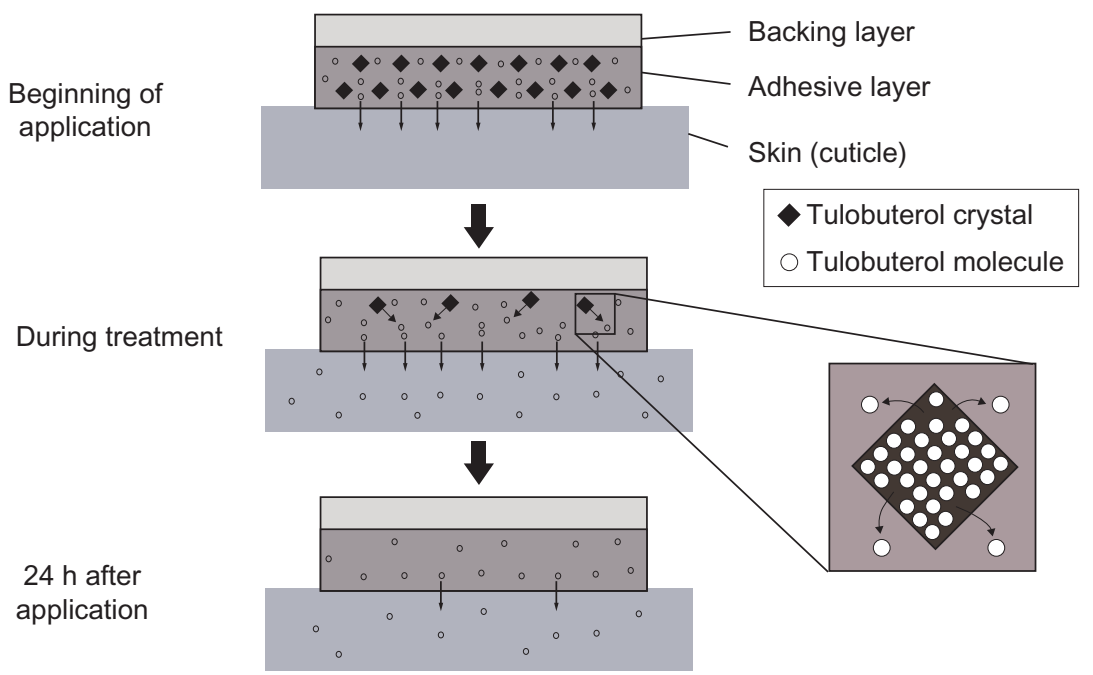

Figure 2 Crystal reservoir system.

Notes: The tulobuterol patch contains crystallized (closed rhombus) and molecular (open circle) forms of tulobuterol in an adhesive layer. When the patch is applied to the skin, molecular tulobuterol is gradually absorbed percutaneously. During application, the number of molecules in the patch is decreased. Subsequently, these molecules are supplied from the crystals.

decreased gradually over the 24 hours following application of the patch. The maximum serum tulobuterol level was $1.4 \mathrm{ng} / \mathrm{mL}$, which is higher than the target effective serum concentration of $1.0 \mathrm{ng} / \mathrm{mL}$. Although tulobuterol was more rapidly absorbed after inhalation, the serum tulobuterol level was maintained in an effective range for a longer period after application of the patch. A mean absorption rate of $90 \%$ was achieved during the 24 hours following application. Mean urinary recovery of unchanged drug after application was $6 \%$. According to the literature, the tulobuterol patch is suitable for preventing the morning dip because peak serum drug levels are achieved during the early morning. Similar pharmacokinetics were observed when the tulobuterol patch was used in children with moderate to severe asthma. ${ }^{10}$ When subjects weighing $<30 \mathrm{~kg}$ received a $1 \mathrm{mg}$ tulobuterol patch and those weighing $\geq 30 \mathrm{~kg}$ received the $2 \mathrm{mg}$ tulobuterol patch, the serum concentration of tulobuterol peaked 12 hours after application and remained at appropriate levels for 24 hours after application. The peak plasma tulobuterol level was $1.33 \mathrm{ng} / \mathrm{mL}$ and the time taken to reach this level was 14 hours. Peak expiratory flow was significantly improved after application of the tulobuterol patch and no side effects were reported. Therefore, the tulobuterol patch is also suitable for use in children with asthma.

\section{Dosage and administration}

In general, $2 \mathrm{mg}$ patches are recommended for adults and children aged 9 years and older; the $0.5 \mathrm{mg}$ patches for children aged 6 months to 2 years; and the $1 \mathrm{mg}$ patches for children aged 3-8 years. The patches are applied to the skin on the chest, back, or upper arm once a day. Although the timing of application for the tulobuterol patch is not clearly described and may depend on disease status, it will be most effective for treatment of asthma if applied in the evening or at bedtime.

\section{Clinical efficacy}

Medications for asthma consist of two types, ie, controllers and relievers. Controllers are medications taken daily on a long-term basis to maintain improvement in asthma symptoms and lung function. Anti-inflammatory drugs, such as inhaled corticosteroids (ICS) and long-acting bronchodilators, are included to this category. ICS are the most effective controllers and are the first-choice anti-inflammatory therapy for asthma. Long-acting $\beta_{2}$-agonists (LABAs), including salmeterol and formoterol, are also categorized as controller medications. In the Japanese guideline for prevention and management of asthma, the tulobuterol patch is also categorized as a controller medication. Use of LABAs as monotherapy is not recommended because these medications have no effects on airways inflammation in asthma and could mask underlying inflammation. Therefore, LABAs should be used only in combination with ICS for the management of asthma.

Tamura et al did a multicenter, randomized, double-blind, parallel-group study to investigate the efficacy and safety of the tulobuterol patch in patients with persistent asthma who were already using ICS. ${ }^{11}$ In this study, 239 adult 
patients with asthma who were on treatment with ICS were randomly assigned to receive the $1 \mathrm{mg}$ tulobuterol patch daily ( $n=116)$ or the $2 \mathrm{mg}$ tulobuterol patch daily $(\mathrm{n}=123)$. During the four-week treatment period, mean peak morning expiratory flow rates in both groups were significantly increased from baseline. This increase was significantly greater in the higher-dose group than in the lower-dose group. In both groups, the frequency of use of short-acting $\beta_{2}$-agonists as rescue medication was significantly decreased from baseline, with no statistically significant difference between the two groups for use of short-acting $\beta_{2}$-agonists as rescue medication or in terms of safety. These data suggest that the tulobuterol patch exerts dose-related add-on effects in asthmatic patients receiving ICS.

Although ICS are widely used as first-line controllers in the long-term management of asthma, ICS alone are insufficient when treating patients with moderate to severe asthma. Such patients frequently experience the morning dip. In the Global Initiative for Asthma, a global guideline for the treatment of asthma, LABAs are positioned as the first add-on controllers for ICS because they can significantly improve airflow obstruction and symptoms. ${ }^{12}$ Sustainedrelease theophylline, designed to be released slowly over an extended period of time and leukotriene receptor antagonists are also considered to be beneficial add-on drugs which have anti-inflammatory activity as well as bronchodilating effects. ${ }^{13-16}$ It has been reported that low-dose sustainedrelease theophylline may be useful as a steroid-sparing agent in combination with low-dose ICS for the treatment of patients with asthma. ${ }^{17,18}$ Leukotriene receptor antagonists reduce the dose of ICS required by patients with moderate to severe asthma and improve asthma control in patients who are not well controlled with ICS monotherapy. ${ }^{19,20}$

Hozawa et al investigated the add-on effects of a tulobuterol patch on peak morning expiratory flow, bronchial hyperresponsiveness, and eosinophilic airways inflammation. ${ }^{21}$ Twenty-four patients with asthma already receiving ICS alone were randomized to receive either ICS \pm a $2 \mathrm{mg}$ /day tulobuterol patch for four weeks. Significant improvements in morning peak expiratory flow and bronchial hyperresponsiveness, as well as a reduction in airway eosinophil infiltration, were observed in the group receiving the add-on tulobuterol patch.

Further, the add-on effects of the tulobuterol patch, sustained-release theophylline, and leukotriene receptor antagonists in patients receiving ICS were compared to determine which of these drugs is the most suitable add-on therapy. Sixty-five asthmatic patients already treated with
ICS alone were randomly divided into four treatment groups: ICS only; ICS + tulobuterol patch; ICS + sustained-release theophylline; or ICS + leukotriene receptor antagonist. FEV and peak expiratory flow values increased markedly in the ICS + tulobuterol patch group compared with those in the other three groups after four weeks of treatment. These data suggest that the tulobuterol patch may be the most effective add-on controller for asthmatic patients already receiving ICS.

Horiguchi et al investigated the long-term efficacy of the tulobuterol patch in patients with asthma. ${ }^{22}$ Twenty-four patients with persistent mild to moderate asthma treated with or without ICS used the tulobuterol patch for one year. Peak morning expiratory flow was significantly increased in all patients after six months of treatment with the tulobuterol patch, and this beneficial effect was maintained throughout the study period. This suggests that the tulobuterol patch can be used for the long-term management of asthma.

The efficacy of the tulobuterol patch for the management of childhood asthma has also been investigated. When children with moderate to severe asthma were treated with the tulobuterol patch, peak expiratory flow values were significantly increased after 24 hours compared with values at baseline. ${ }^{10}$ Katsunuma et al investigated whether the tulobuterol patch was effective in suppressing exacerbations of asthma symptoms caused by upper respiratory tract infections. ${ }^{23}$ Eighty children aged six months to three years with persistent mild to moderate asthma who had received anti-inflammatory drugs when symptoms of upper respiratory tract infection occurred were treated with a tulobuterol or placebo patch. Respiratory symptoms in the group treated with the tulobuterol patch resolved more quickly after the onset of upper respiratory tract infection than in the placebo group. The total respiratory symptom score was also significantly lower in the tulobuterol patch group than in the placebo group. These data suggest that the tulobuterol patch can improve lung function and suppress symptoms of asthma related to upper respiratory tract infection in children. Because the tulobuterol patch is simple to use, it is a practical and effective method for treating infants and young children with asthma.

\section{Tulobuterol patch versus inhaled salmeterol for management of asthma}

Salmeterol is an inhaled LABA used widely as a treatment for asthma in combination with ICS. Fujimoto et al sought to determine the best treatment in patients with uncontrolled 
asthma using ICS, concomitant salmeterol, a concomitant tulobuterol patch, or a double dose of ICS. ${ }^{24}$ In this study, only salmeterol added to ICS significantly improved the morning and evening peak expiratory flow and quality of life score. Kobayashi et al performed a crossover study to investigate whether switching from the tulobuterol patch to salmeterol had a beneficial effect on asthma control. ${ }^{25}$ Sixty-four patients with persistent asthma receiving fluticasone propionate $200-800 \mu \mathrm{g}$ daily were first treated with the tulobuterol patch for four weeks and then switched to inhaled salmeterol for the next four weeks. Salmeterol significantly improved the peak evening expiratory flow compared with the tulobuterol patch. Nishiyama et al also performed a crossover trial to compare the clinical efficacy of salmeterol with that of the tulobuterol patch in 54 patients with moderate to severe asthma despite receiving low-dose ICS. ${ }^{26}$ The patients were divided into two groups, one to receive the tulobuterol patch and the other to receive inhaled salmeterol for four weeks. The patients were then switched to the alternative treatment for a further four weeks. Both medications significantly increased mean peak expiratory flow, although the effect of the tulobuterol patch on lung function was less than that of salmeterol. Scores on the St George's Respiratory Questionnaire (SGRQ), an instrument used to assess health-related quality of life, were significantly improved and comparable between the two drugs.

Although these studies indicate that inhaled salmeterol is more effective than the tulobuterol patch for improvement of lung function in asthmatic patients, tulobuterol patch is still widely used as an add-on drug for ICS in Japan due to its ease of use, and is a useful alternative to LABAs.

\section{Tulobuterol patch versus salmeterol for management of COPD}

A LABA is one of the recommended initial pharmacotherapies for the management of stable COPD in the Global Initiative for Chronic Obstructive Lung Disease (GOLD) guidelines ${ }^{27}$ and in the Japanese guidelines for COPD. The tulobuterol patch is listed under LABAs as a medication for COPD in both guidelines. In the randomized, multicenter, parallel-group, comparative BAREC study, Fukuchi et al compared the efficacy and safety of the tulobuterol patch versus inhaled salmeterol for 12 weeks in 92 patients with stable COPD (GOLD stage II and III). ${ }^{28}$ Peak morning and evening expiratory flow rates were significantly improved in both groups throughout the treatment period, with no significant between-group differences. The total SGRQ score at eight weeks was significantly improved at all time points in the tulobuterol patch group but not in the salmeterol group (Figure 3). Further, compliance with the study regimen was significantly better in the tulobuterol patch group than in the salmeterol group. No serious adverse events were observed in either treatment group. These results suggest that the clinical efficacy of the tulobuterol patch is equivalent or superior to that of salmeterol in the long-term management of patients with stable COPD.

Our research group compared the bronchodilatory efficacy of the tulobuterol patch with that of inhaled salmeterol in 11 patients with stable COPD (GOLD stage II and III). ${ }^{29}$ Both the tulobuterol patch and salmeterol improved $\mathrm{FEV}_{1}$, forced vital capacity, and inspiratory capacity from baseline over 24 hours, and the potency of the bronchodilatory effect of salmeterol was about three times greater than that of the tulobuterol patch. Differences in the results for the two treatment groups in this study may have been due to the study design. It has been reported that a single exposure to the tulobuterol patch may have an insufficient bronchodilatory effect because it takes a couple of days to achieve an effective serum drug concentration. Further study is needed to clarify the bronchodilatory effect of the tulobuterol patch in patients with COPD.

\section{Tulobuterol patch versus theophylline for management of COPD}

Theophylline has long been used as a bronchodilator in the treatment of COPD. Because theophylline is less effective and

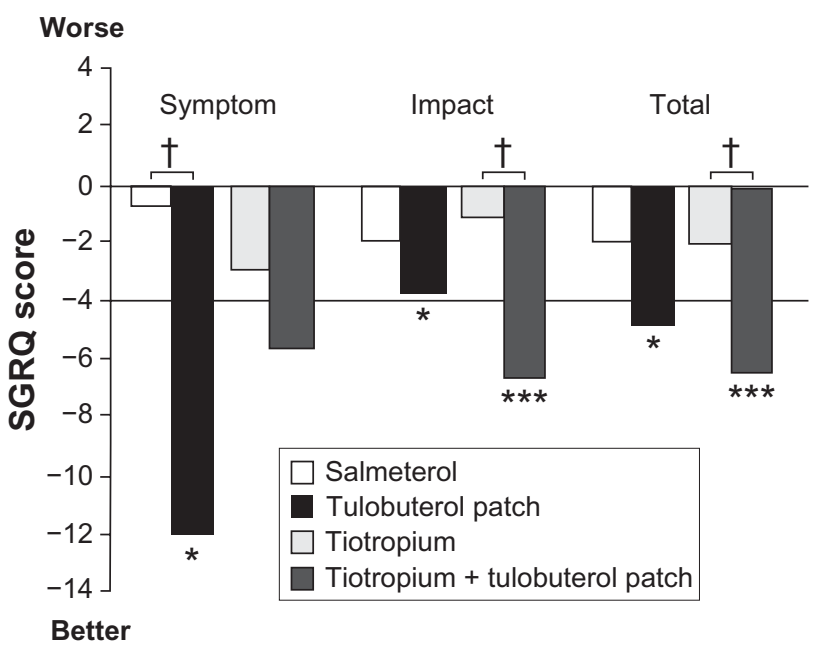

Figure 3 Changes in SGRQ score after 8 weeks of treatment with the tulobuterol patch or salmeterol in patients with stable COPD.

Notes: SGRQ total score, symptom scores, and impact scores were measured at weeks 8 with salmeterol or the tulobuterol patch in patients with COPD, or after use of the tulobuterol patch in patients with COPD receiving tiotropium; changes in the scores from baseline are shown. $* P<0.05$ and $* * * P<0.0001$ versus baseline; t $P<0.05$ tulobuterol patch versus salmeterol.

Abbreviations: COPD, chronic obstructive pulmonary disease; SGRQ, St George's Respiratory Questionnaire. 
less well tolerated than inhaled long-acting bronchodilators, it is recommended as a second-line treatment for COPD in both the GOLD and Japanese guidelines. Theophylline has a weak bronchodilatory effect in stable COPD, ${ }^{30}$ but it has been noted recently that low-dose theophylline has an anti-inflammatory effect in COPD. ${ }^{31}$ Both the transdermally administered tulobuterol patch and oral theophylline are considered to be second-line choices for patients with COPD who cannot use inhaled LABAs or long-acting antimuscarinic agents because of severe pulmonary dysfunction, dementia, or other reasons. A few studies have compared the efficacy of the tulobuterol patch with that of theophylline in COPD. Minami et al reported a randomized, controlled, crossover study comparing the clinical efficacy of the tulobuterol patch and theophylline in patients with stable COPD. ${ }^{32}$ Sixteen patients with stable COPD (GOLD stage I to IV) were randomly allocated to a theophylline group or a tulobuterol patch group for four weeks, and were then switched to the alternative medication for the next four weeks. A significant improvement in symptoms from baseline, including sputum volume, cough frequency, and severity of wheezing, was found with the tulobuterol patch. The SGRQ score was also significantly improved on the tulobuterol patch. These results suggest that the tulobuterol patch is more effective than theophylline for stable COPD. Kanehara et al compared the anti-inflammatory activity and clinical efficacy of low-dose theophylline and the tulobuterol patch in patients with COPD. ${ }^{33}$ In this crossover study, 26 patients with stable COPD (GOLD stage I and II) were randomized to receive theophylline or the tulobuterol patch, with 20 subjects completing the study. Theophylline significantly improved lung function and reduced the number of inflammatory cells in sputum at eight weeks, whereas no significant improvement in lung function was seen in the tulobuterol patch group, and it did not show any antiinflammatory effects. Although many reports have confirmed its bronchodilatory effect in COPD patients, it is unclear why the tulobuterol patch failed to improve the lung function data in this study, and it is possible that the tulobuterol patch may have less anti-inflammatory effect than low-dose theophylline in the airways of COPD patients.

\section{Additive effect of tulobuterol patch in patients already receiving treatment for COPD}

Combined use of two or more long-acting bronchodilators is recommended in the GOLD and Japanese guidelines for the management of stable COPD if symptoms are not improved with monotherapy. A combination of long-acting bronchodilators is preferable to increasing the dose of a single agent in terms of efficacy and adverse effects. $\beta_{2}$-receptors and muscarinic receptors (M3) are both distributed in the central and peripheral airways. Given that vagal nerve fibers mainly innervate the central airways, tiotropium may improve function in the central airways. Further, because the tulobuterol patch distributes the drug more widely throughout the airways via the bronchial and/or pulmonary circulation, extensive bronchodilation of the peripheral airways would be expected with use of the tulobuterol patch as add-on therapy in patients with COPD already being treated with an anticholinergic agent. In this context, combination therapy using the tulobuterol patch and tiotropium could improve obstruction in the central and peripheral airways to a greater extent than anticholinergic monotherapy. Further, improvement of peripheral airways obstruction using the tulobuterol patch may reduce static pulmonary hyperinflation and dynamic hyperinflation during exercise, enabling improved quality of life for patients with COPD. In the multicenter, parallel-group, comparative BAREC II study, Ichinose et al investigated the efficacy and safety of the tulobuterol patch combined with inhaled tiotropium, a longacting anticholinergic agent, in patients with stable COPD. ${ }^{34}$ After a two-week run-in period, 103 patients with stable COPD (GOLD stage II and III) were randomized to receive the tulobuterol patch \pm inhaled tiotropium for eight weeks. Forced vital capacity and $\mathrm{FEV}_{1}$ were significantly improved from baseline and to a similar extent in both groups. However, significant improvement in inspiratory capacity was observed only in the group receiving the tulobuterol patch + inhaled tiotropium. The percentage changes in both peak morning and evening expiratory flow rates significantly increased from one week after the start of treatment, with improvement in peak expiratory flow maintained throughout the study period. The increases in peak morning and evening expiratory flow rates were significantly greater in the tulobuterol patch + inhaled tiotropium group than in the tiotropium only group (Figure 4). A significant improvement in SGRQ score was observed in the tulobuterol patch + inhaled tiotropium group (Figure 3). No serious adverse events occurred in either treatment group.

Our group did a multicenter, randomized, parallel-group study to investigate the additive effect of tiotropium in COPD patients treated with the tulobuterol patch. ${ }^{35}$ Sixty COPD patients receiving the tulobuterol patch were divided into a tiotropium-added group or a tulobuterol patch monotherapy group for four weeks after a two week run-in period. Changes in forced vital capacity and $\mathrm{FEV}_{1}$ were significantly greater 


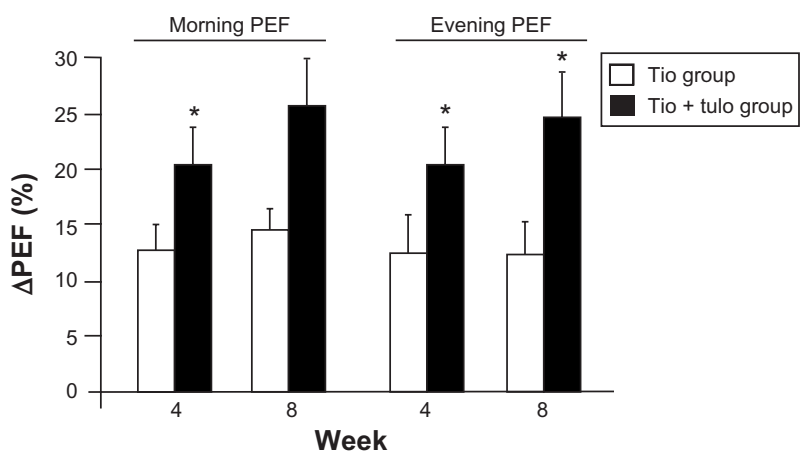

Figure 4 Effect of tulobuterol patch used in combination with tiotropium on peak morning and evening expiratory flow rates.

Notes: Percent changes in peak expiratory flow from baseline are indicated at weeks 4 and 8 after administration of tiotropium or tiotropium with the tulobuterol patch in patients with COPD. Data are expressed as the mean \pm standard deviation. $* P<0.05$, tiotropium versus tiotropium + tulobuterol patch.

Abbreviations: PEF, peak expiratory flow; COPD, chronic obstructive pulmonary disease; Tio, tiotropium; Tulo, tulobuterol patch.

in the tulobuterol patch + tiotropium group than in the tulobuterol patch only group after four weeks of treatment. The percent change in peak expiratory flow was significantly higher in the tulobuterol patch + tiotropium group than in the tulobuterol patch only group after four weeks of treatment. Medical Research Council dyspnea scores were improved only in the tulobuterol patch + tiotropium group. These data suggest that tiotropium could have beneficial effects in patients with COPD receiving the tulobuterol patch.

Further, Abe et al evaluated the effect of inhaled tiotropium + the tulobuterol patch on peripheral airway resistance and reactivity in patients with COPD using impulse oscillation, a forced oscillation technique used to evaluate respiratory resistance and reactivity at various oscillatory frequencies. ${ }^{36}$ Seventeen patients were randomized to receive inhaled tiotropium \pm the tulobuterol patch for four weeks, and were then crossed over to the alternative medication for four weeks. Concomitant treatment with inhaled tiotropium and the tulobuterol patch significantly improved peripheral airways resistance and reactivity assessed by the impulse oscillation system compared with inhaled tiotropium monotherapy. A significant improvement in lung function, including forced vital capacity and $\mathrm{FEV}_{1}$ measured by spirometry, was observed in both treatment groups, but the difference between the two groups did not reach statistical significance. This suggests that the tulobuterol patch can improve peripheral airway obstruction when used in combination with tiotropium.

\section{Treatment adherence}

$\beta_{2}$-agonists can be administered by inhalation, via the oral route, or by transdermal patch for the treatment of asthma and COPD. Because inhaled $\beta_{2}$-agonists are delivered directly into the airways, achieving higher local concentrations and minimizing the risk of systemic adverse events, ${ }^{37}$ inhaled LABAs such as salmeterol are preferred to oral or transdermal $\beta_{2}$-agonists for the long-term management of asthma and COPD. However, elderly and pediatric patients are sometimes unable to inhale these drugs due to insufficient inspiratory muscle force or procedural problems. Further, in the management of chronic disease, adherence with treatment is essential for optimizing the pharmacological efficacy of medication. Therefore, in patients with poor disease status despite use of inhaled drugs, it is necessary to check their inhalation technique and adherence. If they cannot master the devices even after repeated training or show poor adherence with inhaled drugs, alternative medications should be considered. Tamura and Ohta administered an Internet-based questionnaire to patients with asthma or COPD to evaluate their adherence with treatment and the convenience of transdermal and inhaled drug formulations. ${ }^{38}$ Valid answers were obtained from 1470 subjects, including 876 adults with asthma, parents of 171 asthmatic children, and 423 subjects with COPD. The proportion of patients with asthma who took the drugs as indicated was $52.7 \%$ for inhaled agents and $83.2 \%$ for the tulobuterol patch; these proportions were $54.7 \%$ and $86.6 \%$, respectively, for subjects with COPD. The reported rate of adherence was significantly greater for the tulobuterol patch than for any of the inhaled drugs. The most common reason for poor adherence was "frequency of administration". The percentage of tulobuterol patch users who selected "frequency of administration" as their reason for poor adherence was significantly less than that in the subjects who used inhalers. Among the tulobuterol patch users, $79.3 \%$ with asthma and $73.2 \%$ of those with COPD described the tulobuterol patch as "very easy" to use, while the corresponding percentages were $42.7 \%$ and $32.1 \%$, respectively, for users of inhalers. As discussed earlier, the bronchodilatory effect of inhaled salmeterol and tiotropium was superior to that of the tulobuterol patch, but treatment adherence for the tulobuterol patch was among the best for the drugs used in clinical practice. Therefore, the tulobuterol patch can be considered to be very useful for the long-term control of both asthma and COPD.

Mochizuki et al compared adherence rates, effects on exercise tolerance, and quality of life between the tulobuterol patch and inhaled salmeterol in patients with COPD ${ }^{39}$ Forty-four patients with untreated COPD (GOLD stage II and III) were randomly allocated to receive either the tulobuterol patch or inhaled salmeterol for 12 weeks in a crossover manner. The overall adherence rate was $90.3 \%$ for 
the tulobuterol patch and $75.5 \%$ for salmeterol. The rate of adherence with salmeterol was significantly lower in patients aged $\geq 70$ years compared with those aged $<70$ years, whereas the rate of adherence with the tulobuterol patch was favorable across both age groups. Further, adherence with the tulobuterol patch was not affected by cognitive function. The six-minute walk distance was increased after treatment with the tulobuterol patch, and adherence with both medications was positively correlated with changes in the six-minute walk distance. These results show that adherence with the tulobuterol patch was better than with inhaled salmeterol, especially in elderly patients with COPD.

Oral $\beta_{2}$-agonists are another treatment option for such patients. It has been reported that adherence with oral $\beta_{2}$-agonists is better than for inhaled drugs in children with asthma. ${ }^{40}$ However, when tulobuterol is administered orally, the serum concentration of the drug rapidly increases within the first three hours and the duration of effectiveness is shorter than that of inhaled or patch formulations of the drug. Further, systemic side effects are common. Therefore, oral $\beta_{2}$-agonists are described in the Global Initiative for Asthma guideline as being used only on rare occasions when additional bronchodilation is needed. ${ }^{12}$ On the other hand, the tulobuterol patch has the advantage of being easy to use, and the gradual release of tulobuterol provides a stable plasma drug concentration and a long duration of action, which ensures its safety and effectiveness. It is noted in the Japanese guideline for asthma prevention and management that the tulobuterol patch can be a first bronchodilator choice in combination with ICS, especially in infants with moderate to severe asthma. Therefore, the tulobuterol patch might be the best treatment option for pediatric patients with asthma and elderly patients with asthma or COPD.

\section{Safety}

A pharmacokinetic study ${ }^{9}$ reported that tulobuterol released from the patch did not accumulate during repeated transdermal application in healthy adults. It was well tolerated, except for an increase in heart rate of 10-20 beats per minute after five consecutive applications of a $4 \mathrm{mg}$ patch. Further Horiguchi et al reported no signs of tachyphylaxis after use of the tulobuterol patch for a year in adult patients with mild or moderate asthma. ${ }^{22}$ No serious adverse events have been reported in patients with asthma or COPD receiving the $2 \mathrm{mg}$ tulobuterol patch. ${ }^{24,26,28,34,36}$ In these studies, mild adverse events, presumably associated with the patch, were reported as follows: itching, eruption, contact dermatitis, tremor, palpitation, numbness, increased serum creatinine phosphokinase, and abnormal hepatic function. These side effects resolved after stopping the patch. Skin problems related to the tulobuterol patch can be reduced by not applying the patch repeatedly to the same body site. No adverse events were reported in a study of childhood asthma. ${ }^{10,23}$ Another concern related to the use of the tulobuterol patch is that continuous and repeated exposure to $\beta$-adrenoceptor agonists leads to reduced relaxation of smooth muscle in the airways, referred to as desensitization to $\beta$-adrenoceptor agonists. However, Kume et al have reported that chronic exposure to low concentrations of tulobuterol does not lead to desensitization of $\beta$-adrenoceptors on smooth muscle cells in the airways. ${ }^{41}$ Therefore, the tulobuterol patch is well tolerated and can be used for the long-term management of asthma and COPD in any age group.

\section{Conclusion}

The tulobuterol patch with its crystal reservoir system achieves continuous release of tulobuterol for 24 hours after the patch is applied to the skin. Further, serum tulobuterol levels increase gradually and then remain at steady levels. The clinical efficacy of the tulobuterol patch in patients with asthma and COPD has been confirmed by data from several clinical trials. The safety of the tulobuterol patch when used in otherwise healthy adults, children with asthma, and adult patients with asthma or COPD has also been established. It is notable that adherence with treatment is far better in patients using the tulobuterol patch than in those on inhaled drugs. These characteristics of the patch make it useful for long-term management of chronic respiratory disease. The tulobuterol patch might become a first choice in treatment, especially for children and elderly patients who are unable to inhale drugs reliably.

\section{Acknowledgment}

We thank Brent Bell for reading this manuscript.

\section{Disclosure}

The authors declare no conflicts of interest in this work.

\section{References}

1. Burioka N, Miyata M, Endo M, et al. Alteration of the circadian rhythm in peak expiratory flow of nocturnal asthma following nighttime transdermal beta2-adrenoceptor agonist tulobuterol chronotherapy. Chronobiol Int. 2005;22(2):383-390.

2. Kato H, Nagata O, Yamazaki M, Suzuki T, Nakano Y. Development of transdermal formulation of tulobuterol for the treatment of bronchial asthma. Yakugaku Zasshi. 2002;122(1):57-69. Japanese.

3. Turner-Warwick M. Epidemiology of nocturnal asthma. Am J Med. 1988;85(1B):6-8.

4. Martin RJ, Banks-Schlegel S. Chronobiology of asthma. Am J Respir Crit Care Med. 1998;158(3):1002-1007. 
5. Martin RJ, Cicutto LC, Ballard RD. Factors related to the nocturnal worsening of asthma. Am Rev Respir Dis. 1990;141(1):33-38.

6. Hetzel MR, Clark TJ. Comparison of normal and asthmatic circadian rhythms in peak expiratory flow rate. Thorax. 1980;35(10):732-738.

7. Chasseaud LF, Wood SG. Pharmacokinetics of the bronchodilator tulobuterol in man after repeated oral doses. J Int Med Res. 1986; 14(5):223-227.

8. Grassi V, Daniotti S, Schiassi M, Dottorini M, Tantucci C. Oral beta 2-selective adrenergic bronchodilators. Int J Clin Pharmacol Res. 1986 6(2):93-103.

9. Uematsu T, Nakano M, Kosuge K, Kanamaru M, Nakashima M. The pharmacokinetics of the beta 2-adrenoceptor agonist, tulobuterol, given transdermally and by inhalation. Eur J Clin Pharmacol. 1993; 44(4):361-364.

10. Iikura $\mathrm{Y}$, Uchiyama $\mathrm{H}$, Akimoto $\mathrm{K}$, et al. Pharmacokinetics and pharmacodynamics of the tulobuterol patch, $\mathrm{HN}-078$, in childhood asthma. Ann Allergy Asthma Immunol. 1995;74(2):147-151.

11. Tamura G, Sano Y, Hirata K, et al. Effect of transdermal tulobuterol added to inhaled corticosteroids in asthma patients. Allergol Int. 2005; 54(4):615-620.

12. National Heart, Lung and Blood Institute. The Global Strategy for Asthma Management and Prevention, Global Initiative for Asthma 2012. Available from: http://www.ginasthma.org/.

13. Barnes PJ. Theophylline: new perspectives for an old drug. Am J Respir Crit Care Med. 2003;167(6):813-818.

14. Banner KH, Page CP. Theophylline and selective phosphodiesterase inhibitors as anti-inflammatory drugs in the treatment of bronchial asthma. Eur Respir J. 1995;8(6):996-1000.

15. Drazen JM, Israel E, O’Byrne PM. Treatment of asthma with drugs modifying the leukotriene pathway. $N$ Engl J Med. 1999;340(3): 197-206.

16. Lipworth BJ. Leukotriene-receptor antagonists. Lancet. 1999; 353(9146):57-62.

17. Evans DJ, Taylor DA, Zetterstrom O, Chung KF, O'Connor BJ, Barnes PJ. A comparison of low-dose inhaled budesonide plus theophylline and high-dose inhaled budesonide for moderate asthma. N Engl J Med. 1997;337(20):1412-1418.

18. Ukena D, Harnest U, Sakalauskas R, et al. Comparison of addition of theophylline to inhaled steroid with doubling of the dose of inhaled steroid in asthma. Eur Respir J. 1997;10(12):2754-2760.

19. Price DB, Hernandez D, Magyar P, et al. Randomised controlled trial of montelukast plus inhaled budesonide versus double dose inhaled budesonide in adult patients with asthma. Thorax. 2005;58(3):211-216.

20. Vaquerizo MJ, Casan P, Castillo J, et al. Effect of montelukast added to inhaled budesonide on control of mild to moderate asthma. Thorax. 2003;58(3):204-210.

21. Hozawa S, Haruta Y, Terada M, Yamakido M. Effects of the addition of beta2-agonist tulobuterol patches to inhaled corticosteroid in patients with asthma. Allergol Int. 2009;58(4):509-518.

22. Horiguchi T, Kondo R, Miyazaki J, Fukumokto K, Torigoe H. Clinical evaluation of a transdermal therapeutic system of the beta2-agonist tulobuterol in patients with mild or moderate persistent bronchial asthma. Arzneimittelforschung. 2004;54(5):280-285.

23. Katsunuma T, Ohya Y, Fujisawa T, et al. Effects of the tulobuterol patch on the treatment of acute asthma exacerbations in young children. Allergy Asthma Proc. 2012;33(3):e28-e34.

24. Fujimoto K, Komatsu Y, Yasuo M, Urushihata K, Kubo K. Comparison of the clinical efficacy of salmeterol and sustained-release tulobuterol (patch) on inadequately controlled asthma patients on inhaled corticosteroids. J Asthma. 2006;43(7):501-517.
25. Kobayashi Y, Yasuba H, Kudou M, Kita H. Addition of transdermal or inhaled long-acting beta2-agonists in adult asthmatic patients treated with inhaled corticosteroids: switchover study from tulobuterol patch to salmeterol dry powder inhaler. J Asthma. 2007;44(2):77-81.

26. Nishiyama O, Taniguchi H, Kondoh Y, et al. Comparison of the effects of tulobuterol patch and salmeterol in moderate to severe asthma. Clin Exp Pharmacol Physiol. 2006;33(11):1016-1021.

27. [No authors listed]. The Global Strategy for the Diagnosis, Management and Prevention of COPD, Global Initiative for Chronic Obstructive Lung Disease 2011. Available from http://www.goldcopd.org/. Accessed April 10, 2013.

28. Fukuchi Y, Nagai A, Seyama K, et al. Clinical efficacy and safety of transdermal tulobuterol in the treatment of stable COPD: an open-label comparison with inhaled salmeterol. Treat Respir Med. 2005;4(6):447-455.

29. Yamagata T, Hirano T, Sugiura H, et al. Comparison of bronchodilatory properties of transdermal and inhaled long-acting beta 2-agonists. Pulm Pharmacol Ther. 2008;21(1):160-165.

30. Ram FS, Jones PW, Castro AA, et al. Oral theophylline for chronic obstructive pulmonary disease. Cochrane Database Syst Rev. 2002;4: CD003902.

31. Culpitt SV, de Matos C, Russell RE, Donnelly LE, Rogers DF, Barnes PJ. Effect of theophylline on induced sputum inflammatory indices and neutrophil chemotaxis in chronic obstructive pulmonary disease. Am J Respir Crit Care Med. 2002;165(10):1371-1376.

32. Minami S, Kawayama T, Ichiki M, et al. Clinical efficacy of the transdermal tulobuterol patch in patients with chronic obstructive pulmonary disease: a comparison with slow-release theophylline. Intern Med. 2008;47(6):503-509.

33. Kanehara M, Yokoyama A, Tomoda Y, et al. Anti-inflammatory effects and clinical efficacy of theophylline and tulobuterol in mildto-moderate chronic obstructive pulmonary disease. Pulm Pharmacol Ther. 2008;21(6):874-878.

34. Ichinose M, Seyama K, Nishimura M, et al. Additive effects of transdermal tulobuterol to inhaled tiotropium in patients with COPD. Respir Med. 2010;104(2):267-274.

35. Akamatsu K, Yamagata T, Takahashi T, et al. Improvement of pulmonary function and dyspnea by tiotropium in COPD patients using a transdermal beta(2)-agonist. Pulm Pharmacol Ther. 2007;20(6):701-717.

36. Abe T, Setoguchi Y, Kono Y, et al. Effects of inhaled tiotropium plus transdermal tulobuterol versus tiotropium alone on impulse oscillation system (IOS)-assessed measures of peripheral airway resistance and reactance, lung function and quality of life in patients with COPD: a randomized crossover study. Pulm Pharmacol Ther. 2011;24(5): 617-624.

37. Cazzola M, Testi R, Matera MG, et al. Clinical pharmacokinetics of salmeterol. Clin Pharmacokinet. 2002;41(1):19-30.

38. Tamura G, Ohta K. Adherence to treatment by patients with asthma or COPD: comparison between inhaled drugs and transdermal patch. Respir Med. 2007;101(9):1895-1902.

39. Mochizuki H, Nanjo Y, Takahashi H. Better adherence to a transdermal tulobuterol patch than inhaled salmeterol in elderly chronic obstructive pulmonary disease patients. Geriatr Gerontol Int. 2013;13(2):398-404.

40. Ehrlich RI, Du Toit D, Jordaan E, et al. Risk factors for childhood asthma and wheezing. Importance of maternal and household smoking. Am J Respir Crit Care Med. 1996;154(3 Pt 1):681-688.

41. Kume H, Kondo M, Ito Y, Suzuki R, Yamaki K, Takagi K. Effects of sustained-release tulobuterol on asthma control and beta-adrenoceptor function. Clin Exp Pharmacol Physiol. 2002;29(12):1076-1083. 


\section{Publish your work in this journal}

Research and Reports in Transdermal Drug Delivery is an international, peer-reviewed, open access online journal publishing original research, study protocols, reviews, editorials and commentaries on all aspects of transdermal drug delivery. Specific topics in the journal include: Laboratory and clinical development of drug delivery systems including preclinical, clinical studies and protocols; Rationale and basic science; Drug

delivery via gels, creams or patches; Use of chemical drug penetration enhancers ; Patient acceptability studies; and pharmacoeconomic and clinical outcome studies. The manuscript management system is completely online and includes a very quick and fair peer-review system, which is all easy to use. Visit http://www.dovepress.com/ testimonials.php to read real quotes from published authors.

Submit your manuscript here: http://www.dovepress.com/research-and-reports-in-transdermal-drug-delivery-journal 